\title{
Sensitivity Study of Neuronal Excitation and Cathodal Blocking Thresholds of Myelinated Axons for Percutaneous Auricular Vagus Nerve Stimulation
}

\author{
Tom Van de Steene ${ }^{1}$, Emmeric Tanghe ${ }^{1}$, Thomas Tarnaud ${ }^{1}$, Stefan Kampusch ${ }^{2}$, Eugenijus Kaniusas ${ }^{2}$, \\ Luc Martens ${ }^{1}$, Roel Van Holen ${ }^{3}$, Wout Joseph ${ }^{1}$ \\ ${ }^{1}$ Department of Information Technology, Ghent University/imec \\ ${ }^{2}$ Institute of Electrodynamics, Microwave and Circuit Engineering, Vienna University of Technology \\ ${ }^{3}$ Department of Electronics and Information Systems, Ghent University/imec
}

\begin{abstract}
Objective: Excitation of myelinated nerve fibers is investigated by means of numerical simulations, for the application of percutaneous auricular vagus nerve stimulation (pVNS). High sensitivity to axon diameter is of interest regarding the goal of targeting thicker fibers. Methods: Excitation and blocking thresholds for different pulse types, phase durations, axon depths, axon-electrode distances, temperatures and axon diameters are investigated. The used model consists of a $50 \mathrm{~mm}$ long axon and a centrally located needle electrode in a layered medium representing the auricle. Neuronal excitation is simulated using the Frankenhaeuser-Huxley equations for all combinations of parameter values. Results and conclusion: Multiple modes and locations of excitation along the axon were observed, depending on the pulse type and amplitude. When increasing the axonelectrode distance from $1 \mathrm{~mm}$ to $2 \mathrm{~mm}$, sensitivity of thresholds to axon depth decreased with ca. $50 \%$, while sensitivity to axon-electrode distance, axon diameter and phase duration each increased with ca. $15 \%$ to $20 \%$, except from monophasic anodal pulses, showing a $45 \%$ decrease for axon-electrode distance. These trends for axon diameter and axon-electrode distance allow for more selective stimulation of thicker target fibers using monophasic anodal pulses at higher axon-electrode distances. Cathodal monophasic pulses did not perform well due to blocking of the thicker fibers, which was only rarely seen for other pulse types. Significance: Sensitivities of stimulation thresholds to these parameters by numerical simulation reveal how the stimulation parameters can be changed in order to increase therapeutic effect and comfort during pVNS by enabling more selective stimulation.
\end{abstract}

Index Terms-percutaneous auricular vagus nerve stimulation, cathodal blocking, myelinated axon stimulation, parameter sensitivity study

\section{INTRODUCTION AND OBJECTIVES}

$\mathbf{P}$ ERCUTANEOUS auricular vagus nerve stimulation (pVNS) is used as a therapy for refractory epilepsy and chronic pain by stimulation of the auricular branch of the vagus nerve $(\mathrm{ABVN})[1]-[3]$. It has been investigated and further developed as an alternative for invasive vagus nerve stimulation (VNS) during more than a decade, and has the advantage of limited risks for the patient compared to the implantation of a stimulation device during invasive VNS which is, apart from epilepsy, used as a treatment for depression, anxiety, Alzheimer's disease, and migraines [4].

Both, invasive VNS and pVNS stimulate afferent vagus nerve fibers [3], [4]. ABVN fibers are mainly located in the cymba conchae, centrally on the lateral side of the auricle [5]. Small needle electrodes are placed in the auricle in the proximity of $\mathrm{ABVN}$ fibers and the leads are connected to a pulse generator. pVNS has the advantage that needle electrodes can be very precisely placed in the auricle, compared to transcutaneous auricular stimulation in the cymba conchae or tragus (tVNS/taVNS), where much larger skin electrodes are placed on the skin [6], making it a lot more challenging to pinpoint the stimulation region [7]. Nowadays, needle electrodes for $\mathrm{pVNS}$ are placed close to the vessel and nerve branches, without perforating them, typically at a distance of 1 $\mathrm{mm}$ [7]. However, more thorough investigation of the influence of electrode placement might lead to improved insight in the neuronal response, and therefore lead to improved therapy.

This insight of stimulation parameters on the neuronal activation is especially important due to the different fibers that ABVN consists of, and their varying therapeutic effect. Neurons can be classified as A-, B- or C-fibers, each having specific characteristics and functions [8]. For pVNS, therapeutic effect is believed to be mediated by stimulation of (myelinated) $\mathrm{A} \beta$-fibers, a subclass of A-fibers, which are relatively thick, having axon diameters between $7 \mu \mathrm{m}$ and $12 \mu \mathrm{m}[9]$ and therefore are fast-conducting and easily excitable. Auricular (myelinated) $\mathrm{A} \delta$-fibers, having diameters below $6 \mu \mathrm{m}$, and the even smaller (unmyelinated) C-fibers are harder to excite and responsible for the sensation of pain, and therefore stimulation of the latter is not wanted [9], [10]. Due to variations in axon diameter and excitability, extracellular stimulation thresholds can vary for different fiber types as well, which leaves the possibility to selectively stimulate a certain fiber type [9], [11].

Insight in the mechanisms of excitation and blocking of myelinated axons is provided by the activating function [12]. The activating function predicts the locations along an axon where depolarization and hyperpolarization will occur for a certain stimulus and is proportional to the spatial derivative 
of the component of the external electric field along the axon resulting from the stimulus [13]. Depolarization of the axon membrane will facilitate excitation, while hyperpolarization will have the opposite effect. The activating functions for a straight axon and a single electrode close to the middle of the axon are shown for an anodal and a cathodal stimulus in Fig. 1. For an anodal stimulus above the excitation threshold, action potentials will be generated at the two positive lobes in Fig. 1(a), which can propagate towards the respective terminals of the axon. For cathodal stimuli slightly above the excitation threshold, an action potential is generated at the middle of the axon, which will propagate in both directions simultaneously, since the positive peak in Fig. 1 (b) is much higher than the negative lobes at the sides. If the stimulus amplitude is further increased, up to the so-called blocking threshold, the negative lobes will become stronger as well, and hyperpolarization can prevent centrally initiated action potentials from propagating. Consequently, no action potential will reach the brain. As a result, suprathreshold stimulation will be less effective than expected. In practice, the excitation will provide a minimum stimulation amplitude in order to produce an action potential, while the blocking threshold will impose an upper limit on this amplitude (Fig. 11(b)).

The goal of this study is to assess the influence of different parameters on the excitation and blocking threshold for pVNS by means of numerical stimulation. Although the influence of different parameters, including temperature, electrode position, axon diameter, and pulse type, has already been investigated by [14]-[16], the novelty of this study lies among other things in the fact that parameters are varied simultaneously, giving the possibility to investigate interaction between parameters. This is particularly useful to study how dependency on axon diameter varies for changes of other parameters. Subsequently, results could propose how stimulation parameters should be changed in order to maximize stimulation of $\mathrm{A} \beta$-fibers compared to $\mathrm{A} \delta$-fibers and therefore maximize therapeutic effect. Also, apart from excitation thresholds, also blocking thresholds are assessed for the first time. Since thicker fibers are blocked more easily, quantification and prevention of blocking is important for pVNS in order to obtain successful stimulation. Lastly, the model in this study is designed to represent the thickness of a typical human auricle and a realistic electrode model is used in order obtain results that

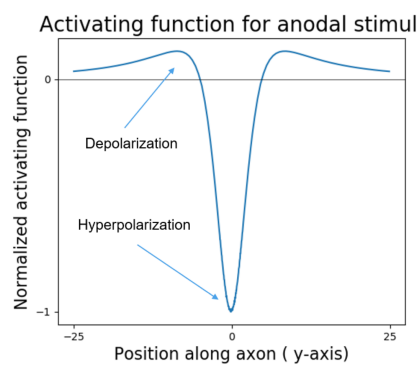

(a)

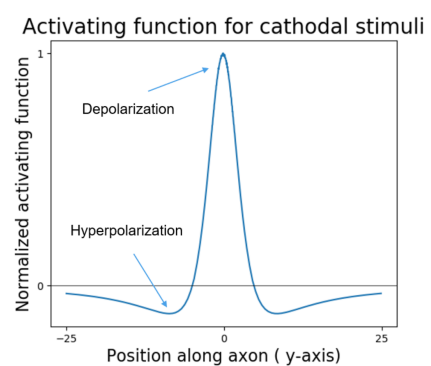

(b)
Fig. 1. (a) Anodal and (b) Cathodal activating function for a straight axon and point current source are representative for the application of $\mathrm{pVNS}$.

In addition to stimulation of $\mathrm{A} \delta$-fibers, stimulation of $\mathrm{C}$ fibers has to be avoided as well. However, since these are unmyelinated fibers, with even higher stimulation thresholds than these of myelinated fibers, a different neuron model would have to be used, complicating the comparison between different fiber types. Consequently, this study is limited to the investigation of myelinated fibers.

\section{Models And Methods}

\section{A. 3D numerical model}

In this study, the auricle is modeled as a flat piece of tissue, consisting of multiple skin, cartilage and subcutaneous fat layers. The thickness of $4 \mathrm{~mm}$ is based on cross-sectional images of an auricle in [17]. The shape of the tissue is taken cylindrical and the needle electrode is placed along its axis in order to obtain an axisymmetric model which is as generic as possible. The thickness of $4 \mathrm{~mm}$ of the tissue corresponds to the height of the cylinder. The diameter of the cylinder is $80 \mathrm{~mm}$. The skin is modeled by three layers, the stratum corneum, cellular epidermis and dermis, with conductivities of $0.0002 \mathrm{~S} / \mathrm{m}$ [18], $0.2 \mathrm{~S} / \mathrm{m}[18]$ and $0.43 \mathrm{~S} / \mathrm{m}[19]$ respectively. On the lateral side of the auricle, in which the needle electrode is placed, a skin layer is present without subcutaneous fat layer. Below the skin is a cartilage layer with conductivity $0.2 \mathrm{~S} / \mathrm{m}$ [20]. At the medial side of the auricle, a skin layer and subcutaneous fat layer with conductivity $0.15 \mathrm{~S} / \mathrm{m}[19]$ are present [21]. The model and thickness of each layer are shown in Fig. 2.

A straight axon of length $50 \mathrm{~mm}$, which is assumed to be considerably longer than typical distances between axon and electrode, is placed in the tissue. The axon is placed parallel to the skin at a certain depth, such that its center perpendicular goes through the electrode. This preserves reflectional symmetry through a plane containing the axis of the cylinder and the middle of the axon. Both the axon depth and axon electrode distance, as measured along the center perpendicular of the axon, are varied. The exact axon length will vary around $50 \mathrm{~mm}$, depending on its internodal length, and therefore on its diameter as described in $\amalg-\mathrm{C}$

\section{B. Simulation software and methodology}

The simulations are performed in two steps, both using the Sim4Life software [22].

First, the electric field in the tissue is calculated for a potential of $1 \mathrm{~V}$ at the electrode. The upper and lower surface of the tissue, the skin, is considered to be in contact with air. A potential of $0 \mathrm{~V}$ is imposed on the side of the cylinder. This methodology preserves the symmetry of the model, with the needle being the axis of symmetry; axons are considered not to have a substantial influence on their surrounding electric field [23]. The current injected by the needle electrode is calculated from the resistance of the tissue between the electrode and the side of the cylinder. The potential distribution along the axon will then be independent of the simulation domain size (cylinder diameter), for a given electrode current. The diameter of the simulation domain of $80 \mathrm{~mm}$ was chosen large enough 


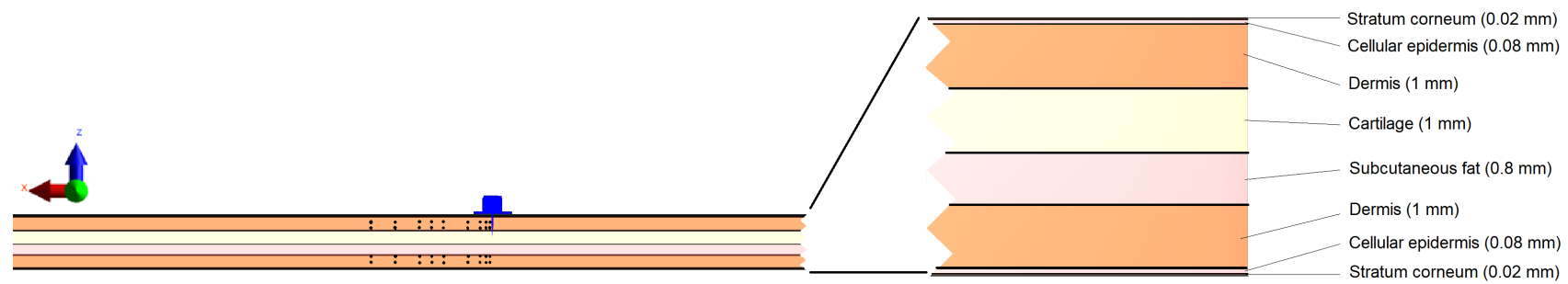

Fig. 2. Cross-section of the cylindrical, numerical simulation model, perpendicularly to the axons, showing tissue layers, needle electrode and axons (black dots, along y-axis). The stratum corneum and cellular epidermis layers are very small and therefore almost indistinguishable in the figure. Layers and thickness are derived from [17], [18], [21]. All combinations of axon-electrode distances and axon depths are shown.

in order not to have a z-component of the electric or current density field at the boundary, Fig 2

The resulting potential distribution is calculated using the Ohmic Electro-quasistatic Solver of the software [22], justified by the relatively low frequencies of stimulation and high conductivity, compared to the electric permittivity. At the frequencies of the relevant harmonic content of the pulses, no reflection or refraction is expected.

Secondly, the potential distribution is used as the input for the neuronal simulations. This field is modulated by a block pulse with a certain amplitude. Subsequently, the built-in neuron solver calculates the response of the axon to the stimulating pulse in terms of the membrane potential along the axon as a function of time.

\section{Neuron model}

The axon is modeled by the spatially extended nonlinear node model (SENN-model) [24] with the equations of Frankenhaeuser and Huxley [25] (FH), describing the membrane characteristics for myelinated axons. The model details are adopted from [24], [26], including that myelin conductance along the internode is neglected and that the internodal distance is defined as 100 times the axon diameter. This implies that the number and location of nodes of Ranvier will vary with the axon diameter. To retain consistency and symmetry for different axon diameters, one node of Ranvier will always be located at the middle of the axon, closest to the electrode. At both sides of the middle, the same number of nodes is added such that the axon length is as close as possible to $50 \mathrm{~mm}$. This means that the axon length will always be an even multiple of the internodal length. The original Frankenhaeuser-Huxley equations are measured at $20^{\circ} \mathrm{C}$, but a correction for other temperatures is done using Q10-values, as described in [27]. This method is used by the software as well to simulate stimulation at different temperatures.

\section{Sensitivity study}

1) Parameter grid: A multitude of parameters are known to have an influence on the stimulation of axons. In this study, the parameters below are varied in their corresponding ranges. Every combination of parameter values is simulated, in order to be able to investigate interaction between multiple parameters. A particular combination of values for each of the parameters is called a case. Parameter ranges, electrode shape, and fiber diameters are especially chosen to be representative and useful for the application of pVNS.

- Electrode - axon distance:

Currently, electrodes are placed at ca. $1 \mathrm{~mm}$ from ABVN in the auricle [7]. In this study, the distance is varied from $0.2 \mathrm{~mm}$ to $10 \mathrm{~mm}$ in order to assess sensitivity to distance over a broad range. The axon is placed at 0.2 - 0.5 - 1 - 2 - 5 - $10 \mathrm{~mm}$. For anodal monophasic and biphasic stimuli with phase duration $0.3 \mathrm{~ms}$, thresholds at $4-6-8 \mathrm{~mm}$ are calculated as well in order to obtain a more uniform distribution for the construction of figures in the discussion section. The value of $0.3 \mathrm{~ms}$ is the longest of the investigated phase durations for which no cathodal blocking was seen during the simulations for the mentioned pulse types. Although electrodes are usually not placed this far, a broad range was chosen to obtain a complete view of the relationship between distance and excitation. Thereby, other nerves in the neighborhood can be present as well, apart from the target nerve.

- Axon depth:

Nerves are expected at depths between $0.5-1 \mathrm{~mm}$ underneath the skin surface, at both sides of the auricle as illustrated in [7]. Considering the thickness of $4 \mathrm{~mm}$, axons at four depths, $0.5-1-3-3.5 \mathrm{~mm}$, are investigated. Fibers of ABVN are found on the lateral side of the auricle, while results for larger axon depths will reveal the possibility of stimulation of axons on the rear side of the auricle.

\section{- Pulse type:}

Both anodal and cathodal stimuli are investigated for monophasic as well as biphasic pulses. In this study, an anodal biphasic pulse is defined as a biphasic pulse with two equal phases, of which the first phase is anodal. Therefore, 4 pulse types in total are investigated. The naming is illustrated in Fig. 3, with normalized amplitude and phase duration.

- Phase duration:

The values of the investigated phase durations in Fig. 3 cover most common clinically used values and are approximately logarithmically distributed, with values at $0.1 \mathrm{~ms}, 0.3 \mathrm{~ms}$, and $1 \mathrm{~ms}$.

- Axon diameter: Varied from $2 \mu \mathrm{m}$ to $12 \mu \mathrm{m}$ in steps of $2 \mu \mathrm{m}$, where diameters up to $6 \mu \mathrm{m}$ represent $\mathrm{A} \delta$-fibers, and larger diameters represent $\mathrm{A} \beta$-fibers.

- Temperature: 

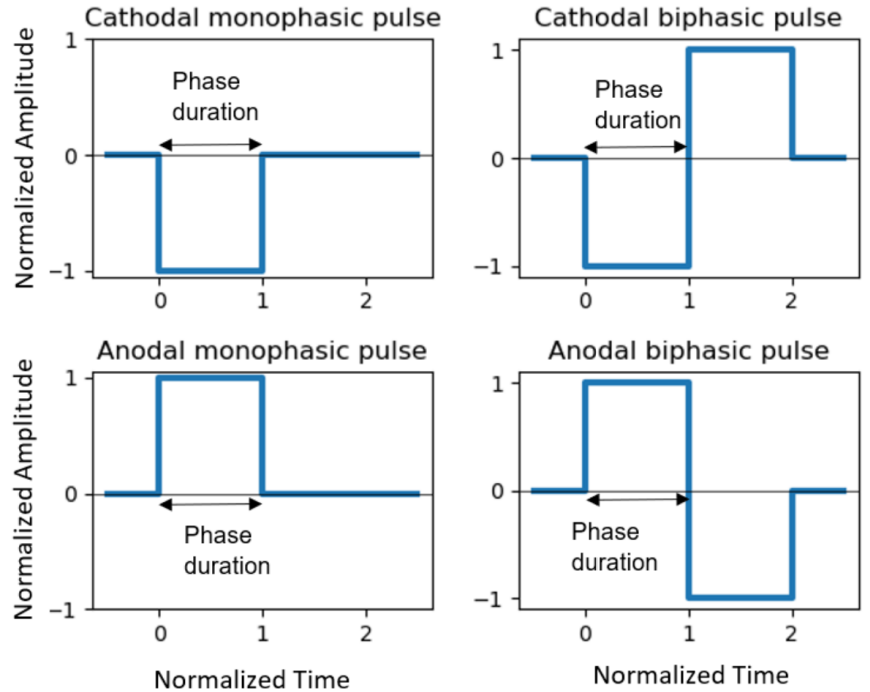

Fig. 3. Overview of the investigated pulse types, showing the used convention for the phase duration and naming of biphasic pulses.

Simulations are carried out for temperature values of 30 , $32,34,36,38$ and $40{ }^{\circ} \mathrm{C}$. However, the effect of temperature on axonal excitation thresholds is already studied by [28] and turned out to be small compared to the influence of other parameters. At supra-threshold amplitudes on the other hand, the effect can become more prominent as described by [29]. To limit computation time, all six temperature values are simulated at a selection containing the most important values for the other parameters. A default value of $36{ }^{\circ} \mathrm{C}$ is used for all other results.

It should be noticed that some of the parameters can indeed be chosen freely during stimulation, whereas others are already fixed: i.e. sensitivity of thresholds to electrode distance, phase duration, and pulse type can be used in an advantageous way in order to reach the goal of optimal $\mathrm{A} \beta$-fiber stimulation. Sensitivity to depth of axons or to small changes in axonelectrode distance on the other hand is interesting to be able to estimate the variations in neuronal response for variations of these parameters and assess the uncertainty of the stimulation outcome when the exact axon depth is not known or when inaccuracies in electrode placement are present.

2) Threshold detection: For every case, both the excitation threshold and blocking threshold are determined by a titration mechanism. The titration factor is the required amplitude of the pulse waveform to induce an action potential. Since the electric field is simulated for an electrode voltage of $1 \mathrm{~V}$, the needed electrode current can be calculated by dividing the titration factor by the total resistance between the electrode and the $0 \mathrm{~V}$ boundary. The blocking threshold is defined as the lowest amplitude at which an action potential is generated that cannot propagate, due to lateral, hyperpolarized regions of the axon. Consequently, the cathodal blocking threshold is higher than the excitation threshold.

In principle, this methodology will yield valid results for myelinated fibers in general according to the SENNFH-model, however, the focus of this study is on pVNS. Individual anatomies of the auricle and branching of ABVN were not taken into account, due to high variability of the anatomy between individuals, yielding highly variable results which are not representative for a whole population.

3) Sensitivity quantification: The proposed methodology will yield a large number of excitation/blocking threshold values considering the high number of cases. To summarize the trends for each of the parameters, in addition to a visual representation, the variability is calculated as the relative change of the threshold $T$ divided by the relative change of parameter $P_{\mathrm{i}}$ as in (1).

$$
\text { variability }_{\mathrm{i}}=\frac{\frac{d T}{T}}{\frac{d P_{\mathrm{i}}}{P_{\mathrm{i}}}}
$$

This value is calculated around a working point (WP), defined by one value for each of the parameters and chosen at relevant points for pVNS: a phase duration of $0.3 \mathrm{~ms}$, based on typical, clinically used values, an axon diameter of $6 \mu \mathrm{m}$, which is in the middle between thick $\mathrm{A} \beta$-fibers and the thin $\mathrm{A} \delta$-fibers. The axon depth is $1 \mathrm{~mm}$, based on images of [17]. The thresholds are highly dependent on axon-electrode distance, consequently, two different WPs are defined, at $1 \mathrm{~mm}$ and $2 \mathrm{~mm}$ for WP1 and WP2 respectively.

\section{RESULTS}

\section{A. Excitation and blocking in terms of membrane potentials}

Fig. 4 shows the multiple possible responses (modes) that were seen for each of the simulated cases at amplitudes above the excitation threshold. In Fig. 4(a), an action potential, defined as a raise of the membrane potential above $10 \mathrm{mV}$, is generated near the center of the axon, close to the electrode, propagating towards the axon terminals, to which will be referred as 'regular stimulation' in the upcoming sections. For cathodal pulses, as in Fig. 4(a), the location of positive activating function, and therefore the expected location of excitation for regular stimulation, will be at the center of the axon, while excitation will occur slightly away from the center for anodal stimuli. In Fig. 4(b), 'terminal stimulation' is illustrated, which means that axons are stimulated at their terminal(s), in contrast to regular stimulation, and an action potential travels towards the other end of the axon. Thirdly, cathodal blocking can occur, where spiking is observed initially but the action potential cannot propagate towards the terminals of the axon, Fig. 4(c). At amplitudes above the excitation threshold, mainly for anodal and biphasic stimuli, the neural reaction to stimulation can become more complex, Fig. 4(d), and action potentials are generated at multiple locations, either at the terminals or at locations along the axon with positive activating function. Action potentials are prevented from further propagation when they bump into each other, or in some cases at locations of hyperpolarization as well.

Firstly, it was observed that for every case, a titration threshold was found, meaning that the axon can theoretically 


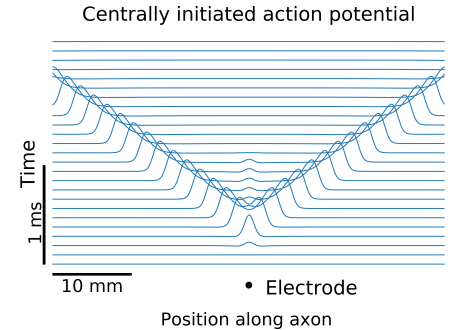

(a)

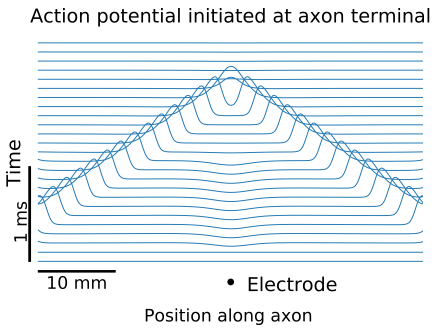

(b)

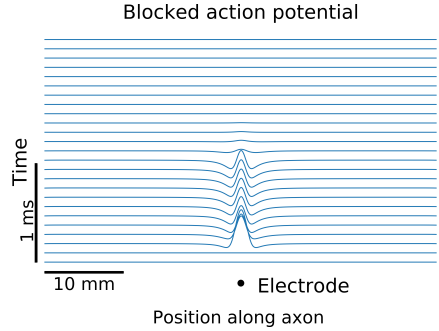

(c)

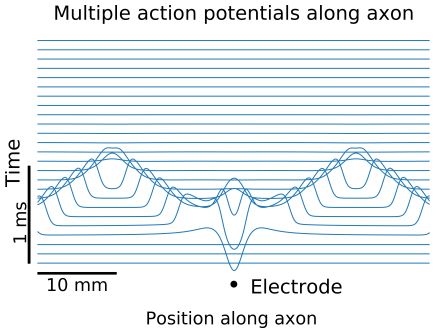

(d)

Fig. 4. Multiple modes of propagation of an action potential along the axon (horizontal axis) at different times (vertical axis). (a) regular stimulation by cathodal stimulus, AP initiated centrally and traveling towards the end of the axon, (b) terminal stimulation, initiated at one end and traveling towards the other end of the axon, (c) AP initiated centrally, but immediately blocked by adjacent hyperpolarized regions and (d) AP initiated at multiple locations along the axon.

always be stimulated, although there will of course be practical limitations on maximum electrode current. At the excitation threshold, either regular or terminal stimulation was observed, corresponding to Fig. 4(a) and (b) respectively.

1) Cathodal monophasic stimuli: axons are stimulated regularly, no terminal stimulation was seen, which is in accordance with the activating function (Fig. 1): the location of positive activating function is always at the middle of the axon. At amplitudes above the excitation threshold, cathodal blocking (Fig 4(c)) occurs, due to locations of negative activating function (Fig. 11.b)). For cathodal monophasic stimuli, blocking thresholds as well as excitation thresholds are reported in III-B.

2) Anodal monophasic stimuli: both regular and terminal stimulation are observed at the excitation threshold, where in general terminal stimulation corresponds to higher axonelectrode distances ( $2 \mathrm{~mm}$ and higher). At amplitudes above the excitation threshold, the response becomes much more complex and less predictable due to multiple excitation and/or blocking regions along the axon. Since no clear blocking as in Fig. 4(c) was observed for anodal monophasic stimuli, no blocking threshold could be determined. Still, the large negative peak of the anodal activating function, can reduce action potentials and sometimes action potentials are prevented from passing the middle of the axon. Considering the left side of the axon as the proximal side, an action potential coming from the right terminal (terminal stimulation) will not be able to induce a therapeutic effect if it is blocked at the location of negative activating function (Fig. 1 (a)). However, for phase durations of $0.3 \mathrm{~ms}$ and shorter, at amplitudes high enough to block an action potential from the right side of the axon, a new action potential is generated at the location of positive activating function (regular stimulation) between the left terminal and the electrode, traveling in both directions such that stimulation is still effective. For phase durations of $1 \mathrm{~ms}$, blocking happened already at lower amplitudes than the generation of the two middle action potentials in Fig. 4(d), and the stimulation could be effectively blocked.

3) Biphasic stimuli: the response is a combination of both situations above. For cases with a relatively low excitation threshold (mainly due to a short axon-electrode distance), the axon is stimulated in a regular way during the cathodal phase of the stimulus and an action potential propagates from the center to the terminals. For cases with higher excitation threshold, terminal stimulation happens more early and the axon is stimulated during the anodal phase. Cathodal blocking as in Fig. 4(c) never occurs, similarly as for anodal monophasic stimuli: for cathodal biphasic stimuli (first phase is cathodal), cathodal blocking can occur at higher amplitudes during this phase. However, during the second, anodal phase an action potential is generated which is able to propagate along the axon.

\section{B. Overview of excitation and blocking thresholds by param- eter}

An overview of all results for the excitation and blocking thresholds is shown in Fig. 5 and Fig. 6, These figures will allow for a qualitative interpretation of the results. Numerical results are given in III-C For each of the pulse types on the left, excitation and blocking thresholds are shown on the $y$-axis. Considering the intended clinical relevance, threshold values higher than $10 \mathrm{~mA}$ are not shown, and are not taken into account for the discussion. For a single row in the figure, every graph shows an identical set of threshold values, although they are classified by a different parameter on the $\mathrm{X}$-axis for every column. Lines in the graphs connect cases for which all parameter values are constant, except from the parameter on the $\mathrm{x}$-axis. This shows the dependency for that particular parameter for each of the cases. Each subfigure (apart from the third column) contains two lines representing an $A \beta$-axon ( $8 \mu \mathrm{m}$, solid line) and an $A \delta$-axon ( $4 \mu \mathrm{m}$, dashed line) at an axon depth of $1 \mathrm{~mm}$, axon-electrode distance of $1 \mathrm{~mm}$, and phase duration of $0.3 \mathrm{~ms}$ of which one parameter is varied. Colors in the figure represent the axon-electrode distance, as illustrated in the second column. However, colors in the figure do not represent vital information and do not change the interpretation of the figure in grayscale. Apart from the figures shown, additional visualization of the data was used to detect trends and draw the conclusions below.

Temperature is not shown in Fig. 5 and Fig. 6, due to the small variation and interaction with other parameters, resulting in a flat course of the thresholds as a function of temperature. The absolute percentage-wise difference of the excitation thresholds between simulations at $30{ }^{\circ} \mathrm{C}$ and $40{ }^{\circ} \mathrm{C}$ was below $20 \%$ for phase durations of $0.1 \mathrm{~ms}$ and became even lower 
than $8 \%$ for phase durations of $1 \mathrm{~ms}$, in contrast to a change of one or more orders of magnitude for some other parameters. Although differences in blocking thresholds between $30{ }^{\circ} \mathrm{C}$ and $40{ }^{\circ} \mathrm{C}$ up to $50 \%$ were possible, this is still much lower than the factors 3 to 100 that are seen between extreme values of other parameters. This observation of higher variability of blocking thresholds compared to excitation thresholds is in accordance with [29]. Due to this relatively low variation, and more importantly, low interaction with other parameters, the upcoming part of the results and discussion comprises simulations at a temperature of $36{ }^{\circ} \mathrm{C}$.

Considering the axon depth (left column of Fig 5), the sensitivity was seen to highly depend on the axon-electrode distance. This can be expected, since a higher sensitivity to axon depth is found for axons close to the electrode, whereas the depth of the axon is of almost negligible influence for axons at higher distances from the electrode. Indeed, the electric field is almost constant over the height of the tissue at relatively high distances from the electrode.

The influence of changing axon-electrode distance is more complex. For monophasic cathodal stimuli, a steadily increasing trend is seen, with a higher sensitivity for more distant axons as well $(2 \mathrm{~mm}$ and more, second graph of the first row in Fig. 55. For biphasic and for monophasic anodal stimuli especially, a transition is seen from an increasing trend for close axons, which abruptly changes to an almost flat course for more distal axons as illustrated in the second column of graphs in Fig. 5. After comparison of these data with the propagation of action potentials for each case, this transition point is identified as the transition from regular to terminal stimulation. Fig. 5 shows that distance dependency is almost lost for terminal stimulation in the investigated range.

The threshold dependency on axon diameter, which is particularly of interest for the application of pVNS, is rather similar for all simulated cases, meaning that the lines of Fig. 5 are mostly parallel, although a more steep trend is seen for the cases with higher thresholds. These mainly correspond to the cases with higher axon-electrode distance.

The thresholds change considerably with changes in phase duration, but since the graphs are again relatively parallel, the interaction with other parameters is limited.

Fig. 6 shows the blocking thresholds for cathodal monophasic stimuli. Higher values of the blocking threshold were not calculated to limit computation time. The trends for the different parameters are qualitatively equal to those of the excitation threshold.

\section{Threshold variability and diameter dependency}

Fig. 5 and Fig. 6 provide a clear idea of the sensitivity to axon depth, axon-electrode distance, axon diameter and phase duration, which can in particular be derived from the slope of the curves. In addition to the visual information from Fig. 5 and Fig. 6, Table I shows the resulting variability for each of the parameters around both WPs, together with the median variability of all cases. Variation of the thresholds for changes in temperature are better expressed as a percentage-wise difference as in III-B above, instead of variability, due to the arbitrariness (in this context) of the $0{ }^{\circ} \mathrm{C}$ reference point of the temperature scale.

At the selected WPs, the phase duration is the least influential parameter, with a variability around -0.2 . The variability for axon depth again illustrates that this parameter becomes less influential at higher axon-electrode distances, with a variability which is two to five times smaller at WP2 $(2 \mathrm{~mm})$ than WP1 $(1 \mathrm{~mm})$.

The variability for axon-electrode distance increases significantly from WP1 to WP2 for cathodal monophasic and for biphasic stimuli (from 1.32 to ca. 1.67), whereas a very high reduction is seen for anodal monophasic stimuli (1.22 to $0.86)$. This reflects the fact that the transition from regular to terminal stimulation occurs at higher distances for biphasic stimuli (ca. $5 \mathrm{~mm}$ ) and is absent for cathodal monophasic stimuli as visible in Fig. 5 .

Values of variability for axon diameter are negative (between -0.95 and -1.39 at the WPs), which is very important, since it confirms that thinner fibers need a higher amplitude to be stimulated. The absolute variability for axon diameter increases as well from WP1 to WP2, which is beneficial considering the goal of selective stimulation of thick fibers.

Table II shows the variabilities of the blocking threshold for cathodal monophasic stimuli. When the variabilities are compared with those for the excitation threshold, a significantly higher value is seen for the blocking threshold (from 1.2 to 2.3 times higher), meaning that the blocking threshold is much more sensitive to changes of the investigated parameters. A way to compare the blocking and the excitation threshold for the cases where both exist, is to look at the ratio of both. This blocking/excitation ratio is found to be between 2.1 and 38, where the lowest ratio is seen for the longest phase duration (which already has the lowest excitation threshold) and the highest ratio is seen for the shortest phase duration (which already has the highest excitation threshold). This is in accordance with the fact that the blocking threshold changes much more heavily for changes in parameters than the excitation threshold. The main influential parameter for the blocking/excitation ratio is the phase duration, suggesting that shorter pulses, or other pulse types than cathodal monophasic pulses, should be used to avoid cathodal blocking.

\section{DisCUSSION}

\section{A. Suprathreshold stimulation and blocking for anodal monophasic and biphasic stimuli}

Compared to monophasic cathodal stimuli, the response to monophasic anodal and biphasic stimuli is more complex and cannot be summarized by the values of excitation and blocking threshold only, since generation and blocking of action potentials happens at different locations along the axon for different stimulus strengths. For phase durations of $0.3 \mathrm{~ms}$ and shorter, action potentials were always able to propagate towards the end of the axon, whereas action potentials were prevented from traveling towards the axon end and the brain for some cases with a phase duration of $1 \mathrm{~ms}$. Consequently, phase durations of $1 \mathrm{~ms}$ are not taken into account for the upcoming discussion. 
Overview of excitation thresholds
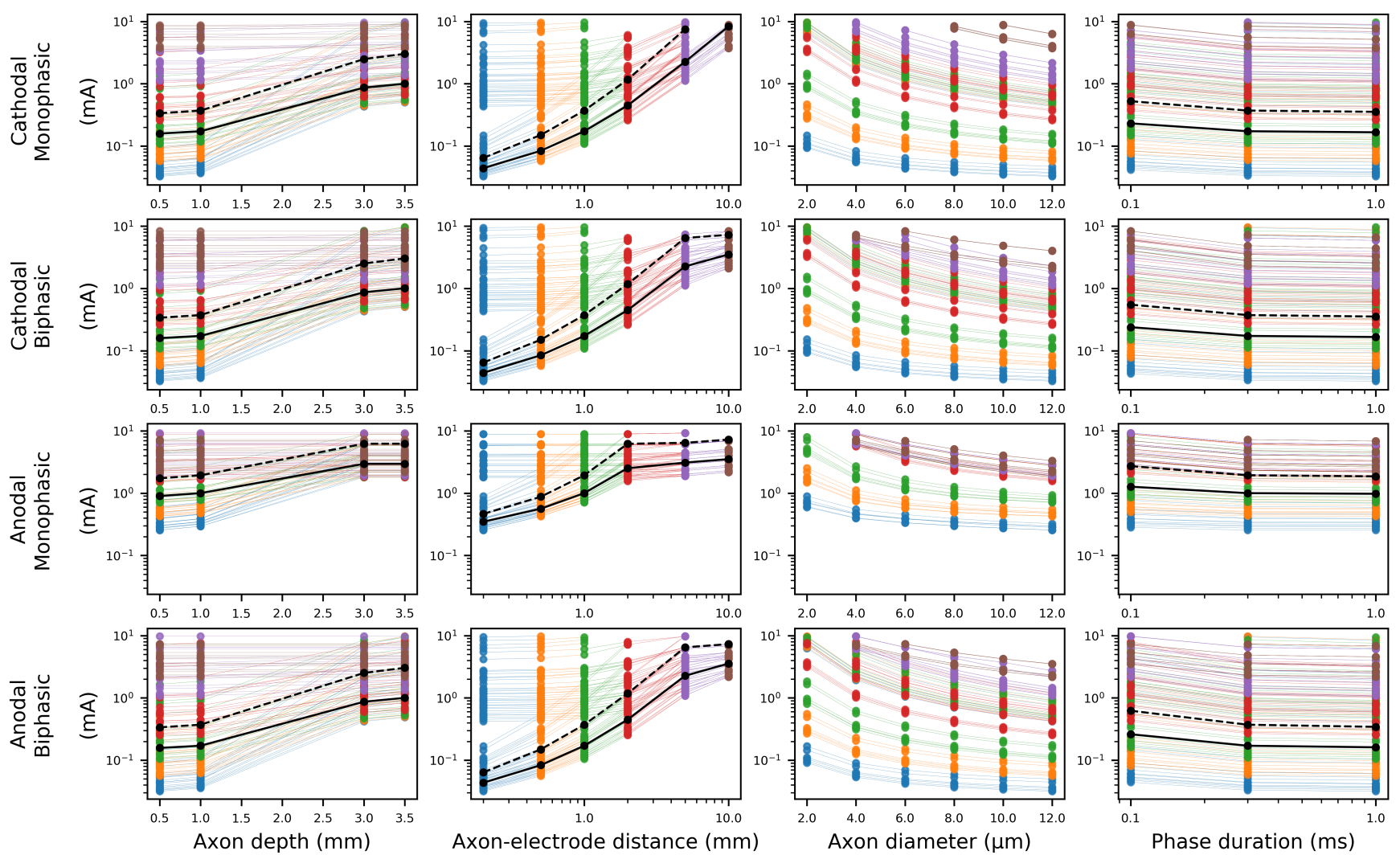

Fig. 5. Overview of all results for different pulse types, cut-off at $10 \mathrm{~mA}$. Parameter values are shown on the x-axis, excitation thresholds on the y-axis). The solid line and the dashed line represent an $\mathrm{A} \beta$-fiber (axon diameter $8 \mu \mathrm{m}$ ) and $\mathrm{A} \delta$-fiber (axon diameter $4 \mu \mathrm{m})$ respectively at an axon depth and axon distance of $1 \mathrm{~mm}$ and phase duration of $0.3 \mathrm{~ms}$. One of these parameters is varied per column. Colors represent the axon-electrode distance as shown in the second column, although the figure can be interpreted without the colors as well.
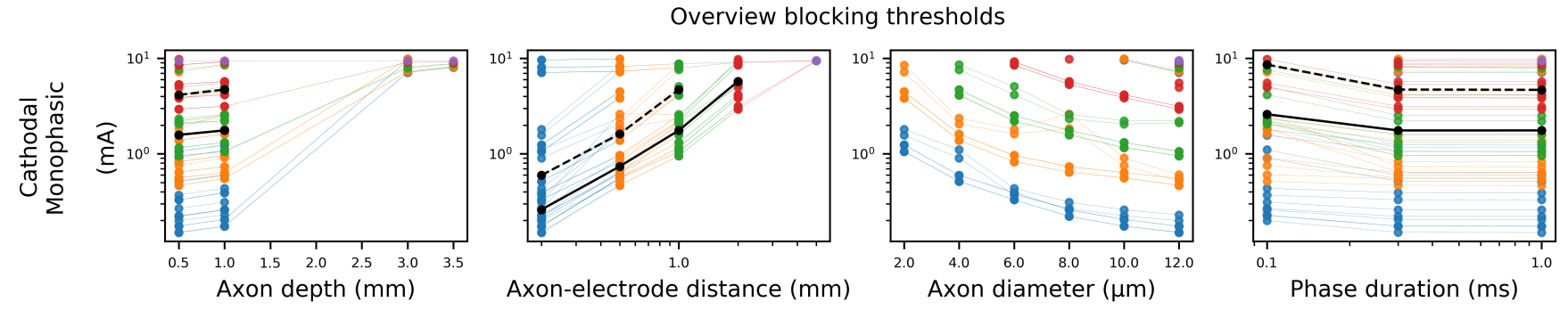

Fig. 6. Overview of blocking threshold for cathodal monophasic pulses, similarly as in Fig. 5

\section{B. Maximum diameter specificity}

Excitation and blocking thresholds depend on a lot of parameters, and targeting large axon diameters in favor of smaller ones, requires an optimal choice of the stimulation parameters. Therefore, it is important to make a distinction between stimulation parameters, which are known and can be set by the physician (pulse type, phase duration, amplitude, approximate axon-electrode distance) and anatomical parameters (axon depth and uncertainty about axon-electrode distance). The latter are only approximately known and actual values are uncertain within a limited range. Optimal therapeutic effect by selective axon diameter stimulation can be achieved by choosing the stimulation parameters in such a way that dependency on axon diameter is maximal, whereas threshold dependency on parameters about which uncertainty exists is minimal. In other words, the cut-off axon diameter of around $6 \mu \mathrm{m}$ should stay relatively constant in a range of axon-electrode distances and axon depths which is as large as possible.

Considering the data in Table I] the variability for axonelectrode distance at WP1 is similar for all pulse types (around 1.3), whereas a very large decrease is seen for anodal monophasic pulses at WP2 (1.22 to 0.86). According to the reasoning above, this lower variability for axon-electrode 
TABLE I

VARIABILITY OF THE EXCITATION THRESHOLDS AROUND WP1 AND WP2

\begin{tabular}{|l|cc|cc|cc|cc|}
\hline \multirow{2}{*}{ Pulse type } & \multicolumn{2}{|c|}{ Axon depth } & \multicolumn{2}{c|}{ Axon-electrode distance } & \multicolumn{2}{c|}{ Axon diameter } & \multicolumn{2}{c|}{ Phase duration } \\
& WP1 & WP2 & WP1 & WP2 & WP1 & WP2 & WP1 & WP2 \\
\hline Cathodal monophasic & 0.34 & 0.19 & 1.32 & 1.67 & -1.10 & -1.38 & -0.15 & -0.18 \\
Cathodal biphasic & 0.34 & 0.19 & 1.32 & 1.67 & -1.11 & -1.39 & -0.17 & -0.20 \\
Anodal monophasic & 0.39 & 0.19 & 1.22 & 0.86 & -0.95 & -1.30 & -0.14 & -0.18 \\
Anodal biphasic & 0.35 & 0.19 & 1.32 & 1.68 & -1.12 & -1.39 & -0.23 & -0.28 \\
\hline
\end{tabular}

TABLE II

VARIABILITY OF THE CATHODAL BLOCKING THRESHOLDS AROUND WP1 AND WP2

\begin{tabular}{|l|cc|cc|cc|cc|}
\hline \multirow{2}{*}{ Pulse type } & \multicolumn{2}{|c|}{ Axon depth } & \multicolumn{2}{|c|}{ Axon-electrode distance } & \multicolumn{2}{c|}{ Axon diameter } & \multicolumn{2}{c|}{ Phase duration } \\
& WP1 & WP2 & WP1 & WP2 & WP1 & WP2 & WP1 & WP2 \\
\hline Cathodal monophasic & 0.50 & 0.29 & 1.63 & - & -1.42 & -1.75 & -0.31 & -0.41 \\
\hline
\end{tabular}

distance allows us to target the thicker fibers in a larger range of distances. Comparing WP1 and WP2 for threshold variability for changes of axon diameter, the increase in absolute value is advantageous as well. Lastly, the axon depth for the WPs is set to $1 \mathrm{~mm}$. For a realistic anatomy however, this value will be uncertain as well. Fortunately, the threshold dependency on axon depth decreases significantly for higher axon-electrode distances (a factor of ca. 2, depending on pulse type), eliminating part of the uncertainty or analogously, increasing the range of depths at which only thicker fibers will be stimulated. Considering these variability values for axonelectrode distance, axon diameter, and axon depth, it might be interesting to use slightly higher axon-electrode distances than the current $1 \mathrm{~mm}$, resulting in higher axon diameter sensitivity, while sensitivities to axon-electrode distance and axon depth are lower. This is also illustrated by the solid and dashed line in Fig. 5 and Fig. 6 . The lines diverge for higher axon-electrode distances, indicating that the difference (on a logarithmic scale) between thick and thin axons increases. A slightly higher difference between both excitation thresholds is seen for shorter phase durations as well. Interestingly, the opposite happens for the blocking thresholds, Fig. 6 . This means that the blocking threshold for thick axons for decreasing phase durations increases faster than for thin axons, which is beneficial in order to maximally stimulate thick, $A \beta$ fibers.

Fig. 7 illustrates how axons are stimulated for different combinations of axon diameter and stimulus amplitude. All axons between $0.2 \mathrm{~mm}$ and $10 \mathrm{~mm}$ are considered. The color of the figure indicates the percentage of stimulated axons if axons with the diameter indicated on the $\mathrm{x}$-axis would be uniformly distributed in this range of distances. Fig. 7 shows the data for the phase duration and axon depth of the WPs, i.e. $0.3 \mathrm{~ms}$ and $1 \mathrm{~mm}$ respectively.

In order to interpret these figures, our goal is expressed as follows. A high capability to target thicker fibers while not stimulating thinner ones, means that at a fixed amplitude, there is a very sharp transition from dark (blue) shades (no or few fibers stimulated) for small axon diameters to bright (yellow) shades (most or all fibers stimulated) for larger axon diameters. This means that the cut-off diameter for stimulation at a certain amplitude is only to a small extent depending

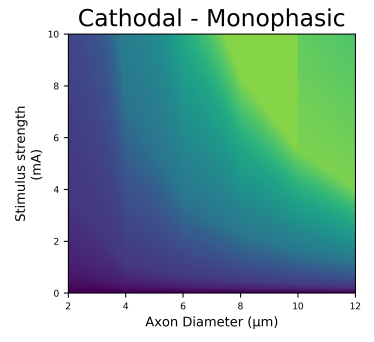

(a)

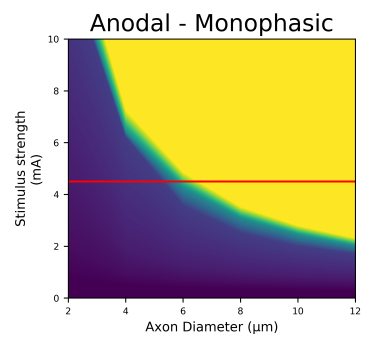

(c)

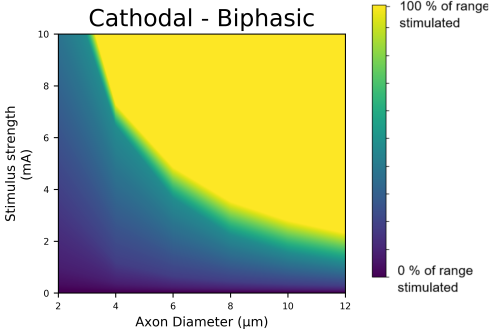

(b)

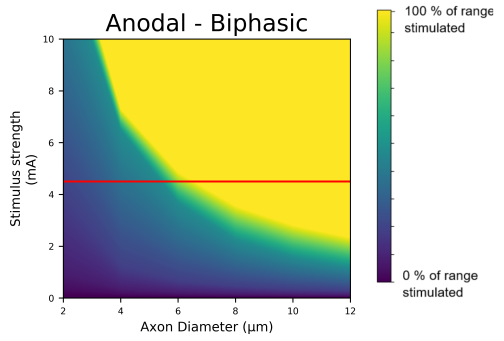

(d)
Fig. 7. Activation of axons in the range of $0.2 \mathrm{~mm}-10 \mathrm{~mm}$ for the axon electrode distance for different pulse types (a) - (d).

on axon-electrode distance. This is visualized by the (red) horizontal line in Fig. 7(c) and (d).

In Fig. 7, axons are considerably less activated for cathodal monophasic stimuli, illustrated by the much darker shade of Fig. 7(a). This is caused by the occurrence of cathodal blocking: at amplitudes high enough to stimulate more distant axons, axons closer to the electrode are already blocked. This means that no amplitude exists at which all axons between $0.2 \mathrm{~mm}$ and $10 \mathrm{~mm}$ are effectively stimulated simultaneously.

Cathodal monophasic stimulation appears to be far from optimal, due to blocking of thicker fibers. Anodal biphasic and cathodal biphasic stimuli on the other hand show very similar results, and for these pulse types and a $0.3 \mathrm{~ms}$ phase duration, no blocking was seen, implying that $100 \%$ activation is always possible. Inspection and analysis of the activation of axons for these pulse types indeed showed that due to the relatively long phase duration of $0.3 \mathrm{~ms}$, excitation was either induced by the anodal or the cathodal phase and was in most cases independent of the order of the two phases, yielding similar excitation thresholds. This is in accordance 
with [30] stating that dependency on the order of the phases is only given for short phase durations, in the order of $100 \mu \mathrm{s}$. Comparing biphasic pulses with anodal monophasic pulses (e.g. Fig. 7(d) vs Fig. 7(c)), a much more gradual transition from dark to bright shades is seen for the former. This indicates that relatively many thin fibers close to the electrode are already stimulated at amplitudes needed for stimulation of more distant, thicker fibers. This implies on its turn that anodal monophasic stimuli are best suited for pVNS, not taking into account that these pulses are not charge balanced. The phase duration mainly changed the scaling of the y-axis, needing higher amplitudes for shorter pulses, but the general shapes of the figures were not significantly altered. This is not the case for a phase duration of $1 \mathrm{~ms}$ however, for which blocking was occasionally observed.

\section{Optimal electrode distance and stimulus amplitude}

Assuming that the approximate location of nerves can be known, e.g. by the use of a finger thimble as in [31], [32], optimal electrode distances and stimulus amplitudes can be extracted from Fig. 8. These figures are derived from Fig. 7(b)(d) and show for each amplitude (x-axis) the cut-off distance (y-axis) for a certain diameter (legend), which is the maximum distance at which axons with that particular diameter will still be stimulated. For the three pulse types in Fig. 8, all axons closer than the cut-off distance from the electrode will be stimulated, while more distant axons will not be stimulated. Obtaining maximum distinction between thin and thick fibers, means finding the window where the difference between these two is maximal. The difference between cut-off distances for $8 \mu \mathrm{m}$ (representing $\mathrm{A} \beta$-axons) and $4 \mu \mathrm{m}$ (representing $\mathrm{A} \delta$-axons) is displayed by the dashed line in Fig. 8(a)-(c). The maximum of this difference is between $1.4 \mathrm{~mm}$ and $10 \mathrm{~mm}$ for anodal monophasic pulses and between $3.3 \mathrm{~mm}$ and $10 \mathrm{~mm}$ for biphasic pulses.

At this maximum, the needed stimulation amplitude turns out to be ca. $3.5 \mathrm{~mA}$ for all pulse types, due to the relatively long phase duration of $0.3 \mathrm{~ms}$ at the WP.

Axons at distances within this range from the electrode will be stimulated if their diameter is $8 \mu \mathrm{m}$ or more, and will not be stimulated if their diameter is $4 \mu \mathrm{m}$ or lower. The exact cutoff axon diameter will be depending on the location within this range.

As shown in Fig. 8, this difference is highest for monophasic anodal stimuli, more specifically at amplitudes between $3 \mathrm{~mA}$ and $6 \mathrm{~mA}$. This implies that instead of placing the electrode at $1 \mathrm{~mm}$ from the axon, which currently is a typical distance, it might be beneficial to place the electrode slightly further than $1.4 \mathrm{~mm}$, e.g. at $2 \mathrm{~mm}$ : if a stimulus of $4 \mathrm{~mA}$ is used, all axons thicker than $6 \mu \mathrm{m}$ are stimulated, while smaller axons are not.

Maximizing this window of distances is important, since in realistic scenarios there will be a lot of variation and uncertainty about stimulation thresholds, exact axon - electrode distance, and other parameters that are currently not taken into account. Biphasic stimuli (Fig. 8(a) and (c)) show higher cutoff distances for thin axons (more than $3 \mathrm{~mm}$ at the maximum

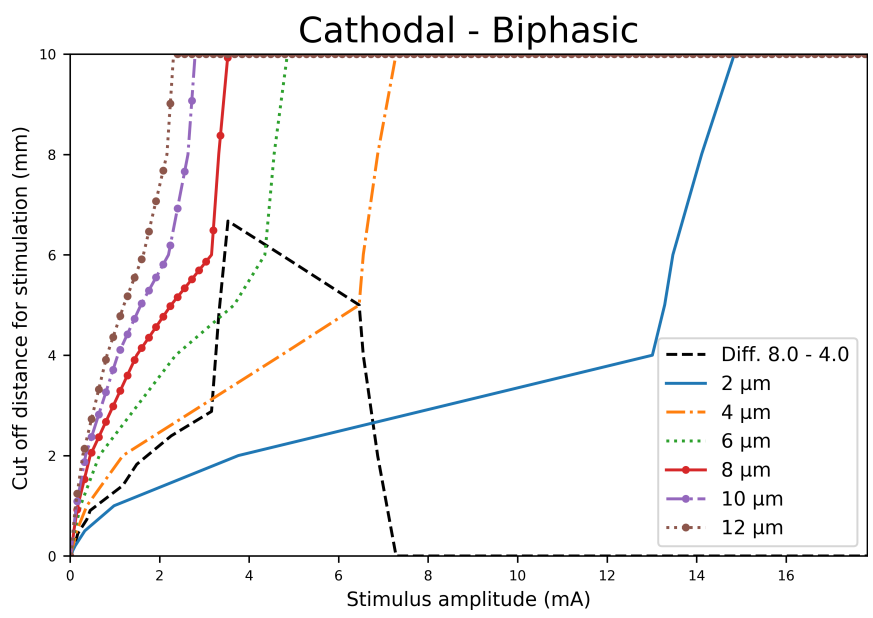

(a) Cathodal Biphasic pulses: Maximum difference between cut-off distances between $3.3 \mathrm{~mm}-10 \mathrm{~mm}$ at amplitude $3.5 \mathrm{~mA}$.

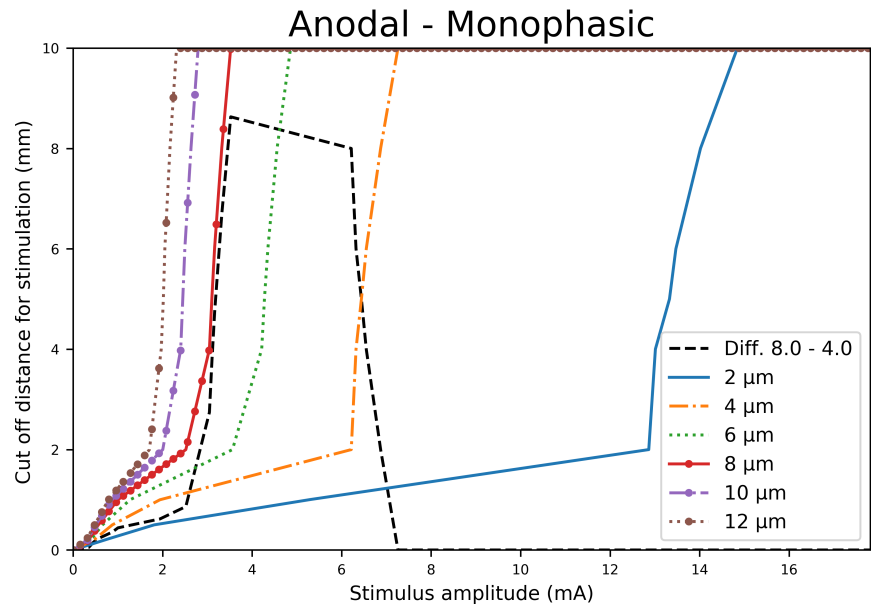

(b) Anodal monophasic pulses: Maximum difference between cut-off distances between $1.4 \mathrm{~mm}-10 \mathrm{~mm}$ at amplitude $3.5 \mathrm{~mA}$.

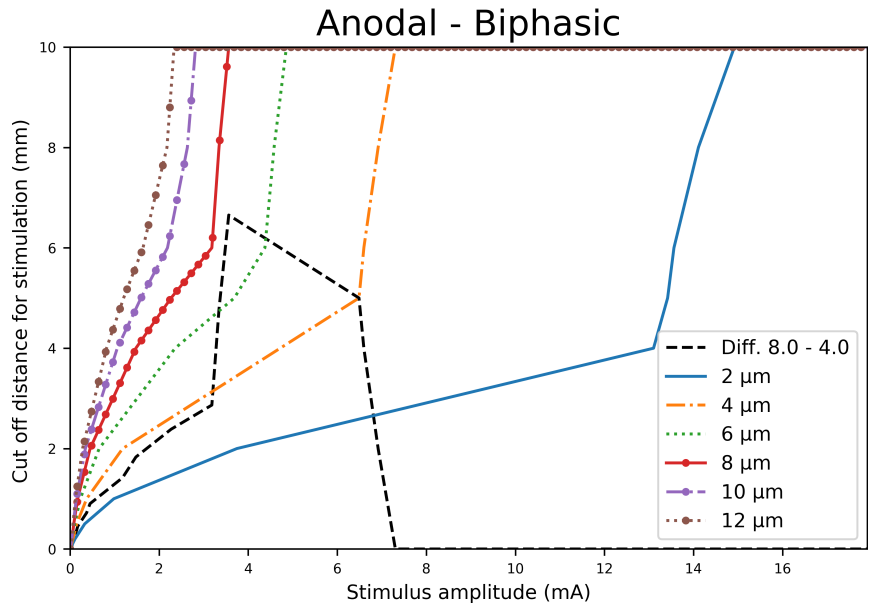

(c) Anodal Biphasic pulses: Maximum difference between cut-off distances between $3.3 \mathrm{~mm}-10 \mathrm{~mm}$ at amplitude $3.6 \mathrm{~mA}$.

Fig. 8. Cut-off distances for stimulation for a series of axon diameters for three pulse types, phase duration of $0.3 \mathrm{~ms}$ and axon depth of $1 \mathrm{~mm}$. 
difference), decreasing the window of optimal distances. This can be explained by the cathodal phase, which stimulates axons close to the electrode at lower amplitudes than the anodal phase.

The results for the maximum difference are of course highly influenced by the maximum axon-electrode distance that was taken into account, being $10 \mathrm{~mm}$. The maximum difference would indeed keep rising otherwise, due to the fast rising cut-off distance of $8 \mu \mathrm{m}$-thick axons. However, this value is chosen since the auricle and cymba conchae can only locally be approximated by a flat piece of tissue, or alternatively, not only the maximum difference is of interest, but keeping the cut-off distance and corresponding amplitude for thin axons reasonably low will be convenient in practice as well.

\section{Validation and Limitations}

The results can be validated by comparison with literature describing both experimental and numerical studies, however, a complete validation of all parameter ranges would not be practically achievable with human subjects due to the large number investigated cases. A recent experimental and numerical pVNS study [30] investigated activation of $\mathrm{A} \beta$ fibers $(8.3 \mu \mathrm{m})$ and $\mathrm{A} \delta$-fibers $(3.5 \mu \mathrm{m})$ for an 'individual numerical model' and a 'simple numerical model', of which the latter corresponds well to this model.

Instead of single axons, multiple axons close to each other are modeled, and relative thresholds for single $\mathrm{A} \beta$-fiber activation (ELa), $100 \% \mathrm{~A} \beta$-fiber activation (ELb) and $50 \% \mathrm{~A} \delta$-fiber activation (ELc) are reported.

A couple of new cases with axon diameter $3.5 \mu \mathrm{m}$ and $8.3 \mu \mathrm{m}$, which correspond to the axon diameters in [30], were simulated for a depth and axon-electrode distance of $1 \mathrm{~mm}$.

Firstly, the ratio of excitation thresholds for both was calculated, which is expected to be in between the ELc/ELb and ELc/ELa ratio for the simple model of [30]. For monophasic pulses, our ratios 2.3 and 2.6 for anodal and cathodal pulses respectively, are in or close to the range of ELc/ELb and ELc/ELa ratios in [30], 2.4 and 2.9 respectively. For biphasic pulses, our ratio of 2.6 turns out to be slightly higher than the ELc/ELa ratio of 2.2 in [30]. Results in this study are compared to in-vivo data in [30] as well, in which three perception levels were defined for each human subject: threshold perception (PLa), comfortable perception (PLb), and the painful up to intolerable perception (PLc). The PLc/PLb amplitude ratios are calculated from the data in Table I in [30], and turned out to be 1.5 for monophasic and biphasic, non-bursted stimulation. This is lower than ratios 2.3 and 2.6. for monophasic and biphasic stimuli respectively in this study. However, this comprises a comparison of single fiber stimulation with experimental perception levels. Compared to our results, PLc levels are lower than expected. This could be related to the higher relative amount of $\mathrm{A} \delta$-fibers in $\mathrm{ABVN}$, facilitating perception by a larger number of stimulated fibers. It is indeed observed by [9] that many more thin $\mathrm{A} \delta$-fibers are present in $\mathrm{ABVN}$ than thick $\mathrm{A} \beta$-fibers. It should be noted as well that high variations are seen for the perception levels PLa, PLb and PLc between subjects, as shown in Fig. 3 in [7], which complicates the deduction of the single value of 1.5 for the ratios of perception levels for comparison with our results.

Secondly, the trends for the investigated pulse types can be investigated. In [30], no significant change between monophasic and biphasic was observed for non-bursted stimulation with a $1 \mathrm{~ms}$ pulse, apart from a $23 \%$ decrease for $\mathrm{A} \delta$ fiber activation in the simple model. In this study, for phase durations of $1 \mathrm{~ms}$, no significant change was seen between cathodal monophasic and biphasic pulses (no distinction is made between anodal and cathodal monophasic pulses in [30]). The significant decrease for $\mathrm{A} \delta$-fibers was not seen either. However, this decrease was also not seen for the experimental data of [30], which is based on the perception levels of the subjects of different stimulus amplitudes.

Furthermore, comparison in [30] of experimental data and numerical results, supports the assumption that $\mathrm{A} \beta$-fiber recruitment can be related to comfortable perception, and $\mathrm{A} \delta$ fiber recruitment to painful perception. This is important since it implies that $\mathrm{A} \delta$-fiber activation can act as an indication of pain perception for the patient.

Lastly, a limited number of locations for the return electrode was investigated in this study as well. For these simulations, a fixed potential of $0 \mathrm{~V}$ was imposed on the return electrode, and the domain boundary was considered electrically isolated instead. Similarly as in [30], the location of the return electrode was changed from $20 \mathrm{~mm}$ to $10 \mathrm{~mm}$, which yielded a change in excitation threshold voltages between $-5.5 \%$ and $-7.5 \%$ for anodal monophasic and biphasic pulses, which corresponds well with the maximum decrease of $-8 \%$ and relative differences up to about $2 \%$ as reported in [30]. The stimulation current on the other hand changed about $2 \%$, which implies that the location of the return electrode does not significantly change the electric field in the proximity of the active electrode for a constant current, but rather influences the 'input impedance' of the electrode setup. Subsequently, the location of the return electrode around the active electrode was changed to 4 different locations in total, while keeping the distance of $10 \mathrm{~mm}$ in the perpendicular and/or parallel direction constant, which showed that only limited changes of up to around $10 \%$ and $20 \%$ in stimulation current were seen at the parameters of WP1 and WP2 respectively. From these results, no significantly optimal location for the return electrode can be indicated.

Validation of the SENN-model with FrankenhaeuserHuxley equations has been carried out by comparison with the model of a rat neuron from Schwarz-Eikhof [33], [34]. Both excitation and blocking thresholds were assessed. Absolute excitation threshold values of the Schwarz-Eikhof model varied up to a factor 2 and blocking thresholds up to a factor 4 w.r.t the SENN-FH-model, but it was seen that qualitative trends were preserved. Figures could be reproduced, although they were shifted to slightly higher amplitudes. Variability values were similar between both models, with maximum variations of around $20 \%$, while also preserving relative differences between pulse types, 
parameters and working points, apart from phase duration, which was up to a factor 4 higher for the Schwarz-Eikhof model [33]. However, the phase duration is a stimulation parameter, which can be exactly set by the physician.

Comparison with a homogeneous model, by setting a constant conductivity of $0.2 \mathrm{~S} / \mathrm{m}$ for all tissue layers also showed that variabilities did not change much. Only a shift to lower stimulation currents was seen for the absolute thresholds, due to the lower average conductivity.

Since variability values were not very sensitive to changes in the geometric model or the neuronal model, they appear to be rather robust, and are not likely to change much between individuals, although a shift of the threshold amplitudes themselves between individuals could be possible on the other hand.

While a lot of parameters are investigated in this study, the axon length was not yet varied in the simulations. Although the current length of $50 \mathrm{~mm}$ is long, compared to the axonelectrode distances which are up to $10 \mathrm{~mm}$, terminal stimulation was still seen, meaning that the axon cannot be considered infinite. Subsequently, the axon length can have in influence on the thresholds as well, and in particular the transition from regular to terminal stimulation. Therefore, future work will include investigation of the influence of axon length and/or investigation of (semi-)infinite axons. Future work also including bent axons instead of straight axons only, might be interesting to investigate the robustness of the variability values. Also, further investigation of how blocking and excitation interact at supra-excitation threshold amplitudes, might provide more insight in the neuronal response, in particular for phase durations of $1 \mathrm{~ms}$, since multiple excitation and/or blocking thresholds could be determined for a selection of cases.

\section{CONCLUSiON}

Excitation and blocking thresholds of myelinated axons for the application of pVNS are investigated, aiming to target the thicker $\mathrm{A} \beta$-fibers. Pulse type, axon-electrode distance, axon depth, axon diameter, and phase duration are simultaneously varied and the variabilities of the thresholds for each of the parameters is calculated. For cathodal monophasic stimuli, cathodal blocking occurs, limiting the potential of effective stimulation of the nerve fibers, and in particular thick fibers. No clear effective blocking was seen for monophasic anodal and biphasic stimuli for phase durations up to $0.3 \mathrm{~ms}$, action potentials occasionally appeared to be blocked for a phase duration of $1 \mathrm{~ms}$.

Considering the variabilities of the parameters, axon depth was of importance at small electrode-axon distances only, with a variability of ca. 0.35 at $1 \mathrm{~mm}$. Phase duration had an influence on how axons are activated and on the needed amplitude, but did not interact considerably with other parameters concerning the value of the thresholds. The variability of the excitation threshold for phase duration is between 0.12 and 0.28 depending on pulse type, for distances between $1 \mathrm{~mm}$ and $2 \mathrm{~mm}$. The variability of the blocking threshold was ca.
1.5 times higher compared to the excitation threshold, inferring that there is a larger stimulation window for shorter phase durations.

The axon-electrode distance was the most interesting parameter revealing two modes of excitation, regular and terminal stimulation, of which the latter occurred for anodal phases only and at higher axon-electrode distances (2 $\mathrm{mm}$ and more) and had a lower variability than regular stimulation. Smaller sensitivity to axon-electrode distance at higher distances is wanted (1.22 at $1 \mathrm{~mm}$ vs 0.86 at $2 \mathrm{~mm}$ for anodal monophasic stimuli), in order to target only thick fibers in a region as large as possible.

This, combined with a higher variability of the excitation threshold for changes in diameter $(-0.95$ up to -1.30 at $2 \mathrm{~mm}$ ), suggests that placing electrodes at slightly higher distances (e.g. $2 \mathrm{~mm}$ instead of $1 \mathrm{~mm}$ ) could improve the therapeutic outcome by optimal stimulation of thicker A $\beta$-fibers, while limiting pain due to stimulation of thinner $\mathrm{A} \delta$-fibers. However, reaching a larger area of stimulation of thick ABVN fibers can come at the cost of increased stimulation of non-ABVN fibers as well. The highest selectivity could be achieved with monophasic anodal pulses, which are unfortunately not charge-balanced.

The variability values, on which these conclusions are based, showed reasonable robustness for changes to both the geometric and neuronal model, although individual threshold amplitudes are more likely to vary between individuals. Future work will focus on dependency on axon geometry, including axon length and shape.

\section{ACKNOWLEDGMENT}

This work was supported by the Research Foundation Flanders (FWO-V) under grant agreement No G003415N and the Austrian Research Promotion Agency.

\section{REFERENCES}

[1] S. Bauer, H. Baier, C. Baumgartner, K. Bohlmann, S. Fauser, W. Graf, B. Hillenbrand, M. Hirsch, C. Last, H. Lerche, T. Mayer, A. Schulze-Bonhage, B. Steinhoff, Y. Weber, A. Hartlep, F. Rosenow, and H. Hamer, "Transcutaneous Vagus Nerve Stimulation (tVNS) for Treatment of Drug-Resistant Epilepsy: A Randomized, Double-Blind Clinical Trial (cMPsE02)," Brain Stimulation, vol. 9, no. 3, pp. 356363, may 2016. [Online]. Available: https://linkinghub.elsevier.com/ retrieve/pii/S1935861X15012255

[2] S. Sator-Katzenschlager, J. Szeles, G. Scharbert, A. Michalek-Sauberer, A. Kober, G. Heinze, and S. Kietaibl, "Electrical Stimulation of Auricular Acupuncture Points Is More Effective Than Conventional Manual Auricular Acupuncture in Chronic Cervical Pain: A Pilot Study,', Anesthesia and analgesia, vol. 97, pp. 1469-1473, 2003.

[3] E. Kaniusas, S. Kampusch, M. Tittgemeyer, F. Panetsos, R. F. Gines, M. Papa, A. Kiss, B. Podesser, A. M. Cassara, E. Tanghe, A. M. Samoudi, T. Tarnaud, W. Joseph, V. Marozas, A. Lukosevicius, N. Ištuk, A. Šarolić, S. Lechner, W. Klonowski, G. Varoneckas, and J. C. Széles, "Current Directions in the Auricular Vagus Nerve Stimulation I - A Physiological Perspective," Frontiers in Neuroscience, vol. 13, p. 854, aug 2019. [Online]. Available: https://www.frontiersin.org/article/10.3389/fnins.2019.00854/full

[4] D. A. Groves and V. J. Brown, "Vagal nerve stimulation: a review of its applications and potential mechanisms that mediate its clinical effects," Neuroscience \& Biobehavioral Reviews, vol. 29, no. 3, pp. 493-500, may 2005. [Online]. Available: https://linkinghub.elsevier.com/retrieve/ pii/S0149763405000199 
[5] E. T. Peuker and T. J. Filler, "The nerve supply of the human auricle," Clinical Anatomy, 2002.

[6] B. Badran, A. Yu, D. Adair, G. Mappin, W. DeVries, D. Jenkins, M. George, and M. Bikson, "Laboratory Administration of Transcutaneous Auricular Vagus Nerve Stimulation (taVNS): Technique, Targeting, and Considerations," J. Vis. Exp, p. 58984, 2018. [Online]. Available: www.jove.comurl:https://www.jove.com/video/58984

[7] E. Kaniusas, S. Kampusch, M. Tittgemeyer, F. Panetsos, R. F. Gines, M. Papa, A. Kiss, B. Podesser, A. M. Cassara, E. Tanghe, A. M. Samoudi, T. Tarnaud, W. Joseph, V. Marozas, A. Lukosevicius, N. Ištuk, S. Lechner, W. Klonowski, G. Varoneckas, J. C. Széles, and A. Šarolić, "Current Directions in the Auricular Vagus Nerve Stimulation II An Engineering Perspective," Frontiers in Neuroscience, vol. 13, p. 772, jul 2019. [Online]. Available: https://www.frontiersin.org/article/ 10.3389/fnins.2019.00772/full

[8] H. S. Gasser, "The Classification of Nerve Fibers," The Ohio Journal of Science, vol. 41, no. 3, pp. 145-159, 1941. [Online]. Available: https://kb.osu.edu/handle/1811/3153?show=full

[9] S. Safi, J. Ellrich, and W. Neuhuber, "Myelinated Axons in the Auricular Branch of the Human Vagus Nerve," Anatomical Record, vol. 299, no. 9, pp. 1184-1191, 2016.

[10] M. Evans, Verma-Ahuja S, Naritoku DK, and Espinosa JA, "Intraoperative human vagus nerve compound action potentials," Acta Neurol Scand, vol. 110, pp. 232-238, 2004. [Online]. Available: https: //onlinelibrary.wiley.com/doi/pdf/10.1111/j.1600-0404.2004.00309.x

[11] S. L. Helmers, J. Begnaud, A. Cowley, H. M. Corwin, J. C. Edwards, D. L. Holder, H. Kostov, P. G. Larsson, P. M. Levisohn, M. S. De Menezes, H. Stefan, and D. M. Labiner, "Application of a computational model of vagus nerve stimulation," Acta Neurologica Scandinavica, vol. 126, no. 5, pp. 336-343, 2012.

[12] F. Rattay, "Analysis of models for extracellular fiber stimulation," IEEE Transactions on Biomedical Engineering, vol. 36, no. 7, pp. 676-682, jul 1989. [Online]. Available: http://ieeexplore.ieee.org/document/32099/

[13] _ "Ways to approximate current-distance relations for electrically stimulated fibers," Journal of Theoretical Biology, vol. 125, no. 3, pp. 339-349, 1987.

[14] T. Tarnaud, W. Joseph, L. Martens, and E. Tanghe, "Dependence of excitability indices on membrane channel dynamics, myelin impedance, electrode location and stimulus waveforms in myelinated and unmyelinated fibre models," Medical and Biological Engineering and Computing, vol. 56, no. 9, pp. 1595-1613, 2018.

[15] A. M. Samoudi, S. Kampusch, E. Tanghe, J. C. Széles, L. Martens, E. Kaniusas, and W. Joseph, "Numerical modeling of percutaneous auricular vagus nerve stimulation: a realistic 3D model to evaluate sensitivity of neural activation to electrode position," Medical and Biological Engineering and Computing, 2017.

[16] A. Samoudi, S. Kampusch, E. Tanghe, J. Széles, L. Martens, E. Kaniusas, and W. Joseph, "Sensitivity Analysis of a Numerical Model for Percutaneous Auricular Vagus Nerve Stimulation," Applied Sciences, vol. 9, no. 3, p. 540, feb 2019. [Online]. Available: http://www.mdpi.com/2076-3417/9/3/540

[17] P. Bermejo, M. López, I. Larraya, J. Chamorro, J. L. Cobo, S. Ordóñez, and J. A. Vega, "Innervation of the Human Cavum Conchae and Auditory Canal: Anatomical Basis for Transcutaneous Auricular Nerve Stimulation," BioMed Research International, 2017.

[18] V. De Santis, X. L. Chen, I. Laakso, and A. Hirata, "An equivalent skin conductivity model for low-frequency magnetic field dosimetry," Biomedical Physics \& Engineering Express, 2015.

[19] K. Wake, K. Sasaki, and S. Watanabe, "Conductivities of epidermis, dermis, and subcutaneous tissue at intermediate frequencies," Physics in Medicine and Biology, vol. 61, no. 12, pp. 4376-4389, jun 2016. [Online]. Available: http://stacks.iop.org/0031-9155/61/i=12/a= 4376?key=crossref.3fd8b53120fbaa4e8dcbdab55f258ebe

[20] C. Gabriel, "Compilation of the dielectric properties of body tissues at RF and microwave frequencies," Tech. Rep., 1996.

[21] E. Reichman, Emergency Medicine Procedures 2nd edition. McGrawHill, 2013.

[22] Zurich MedTech, "Sim4Life," 2018. [Online]. Available: Zmt.swiss/ sim4life/

[23] R. Plonsey, "The active fiber in a volume conductor," IEEE Transactions on Biomedical Engineering, vol. BME-21, no. 5, pp. 371-381, sep 1974. [Online]. Available: http://ieeexplore.ieee.org/document/4120805/

[24] J. P. Reilly, V. T. Freeman, and W. D. Larkin, "Sensory effects of transient electrical stimulation-evaluation with a neuroelectric model," IEEE Trans Biomed Eng, vol. 32, no. 12, pp. 1001-1011, dec 1985.

[25] B. Frankenhaeuser and A. F. Huxley, "The action potential in the myelinated nerve fibre of Xenopus laevis as computed on the basis of voltage clamp data," The Journal of Physiology, vol. 171, no. 2, pp. 302-315, jun 1964.

[26] D. R. McNeal, "Analysis of a Model for Excitation of Myelinated Nerve," IEEE Transactions on Biomedical Engineering, vol. BME-23, no. 4, pp. 329-337, 1976.

[27] B. Frankenhaeuser and L. E. Moore, "The effect of temperature on the sodium and potassium permeability changes in myelinated nerve fibres of Xenopus laevis," The Journal of Physiology, vol. 169, no. 2, pp. 431-437, 1963.

[28] A. M. Samoudi, "Electromagnetic Modelling and Optimization for SPECT-MRI and Auricular Vagus Nerve Stimulation," Ph.D. dissertation, Ghent University, 2017. [Online]. Available: https: //biblio.ugent.be/publication/8560652/file/8560653

[29] F. Rattay and M. Aberham, "Modeling Axon Membranes for Functional Electrical Stimulation," IEEE Transactions on Biomedical Engineering, vol. 40, no. 12, pp. 1201-1209, 1993.

[30] E. Kaniusas, A. M. Samoudi, S. Kampusch, K. Bald, E. Tanghe, L. Martens, W. Joseph, and J. C. Szeles, "Stimulation Pattern Efficiency in Percutaneous Auricular Vagus Nerve Stimulation: Experimental versus Numerical data," IEEE Transactions on Biomedical Engineering, vol. 9294, no. c, pp. 1-1, 2019. [Online]. Available: https://ieeexplore.ieee.org/document/8889430/

[31] E. Kaniusas, G. Varoneckas, B. Mahr, and J. C. Szeles, "Optic visualization of auricular nerves and blood vessels: Optimisation and validation," in IEEE Transactions on Instrumentation and Measurement, 2011.

[32] B. D. Razlighi, S. Kampusch, S. H. Geyer, V. Hoang Le, F. Thurk, S. Brenner, J. C. Szeles, W. J. Weninger, and E. Kaniusas, "InSilico Ear Model Based on Episcopic Images for Percutaneous Auricular Vagus Nerve Stimulation," in 2018 EMF-Med 1st World Conference on Biomedical Applications of Electromagnetic Fields $(E M F-M e d)$. IEEE, sep 2018, pp. 1-2. [Online]. Available: https: //ieeexplore.ieee.org/document/8526013/

[33] J. R. Schwarz and G. Eikhof, "Na currents and action potentials in rat myelinated nerve fibres at $20^{\circ} \mathrm{C}$ and $37^{\circ} \mathrm{C}$," Pflugers Archiv : European journal of physiology, vol. 409, no. 409, pp. 569-577, aug 1987.

[34] S. Raspopovic, M. Capogrosso, and S. Micera, "A computational model for the stimulation of rat sciatic nerve using a transverse intrafascicular multichannel electrode," IEEE Transactions on Neural Systems and Rehabilitation Engineering, vol. 19, no. 4, pp. 333-344, aug 2011. 